\title{
Performance of Cross-Layer Design with Antenna Selection and Imperfect Feedback Information in MIMO Systems
}

\author{
Xiaoyu Dang, Xiangbin Yu, and Xiaomin Chen \\ College of Electronic and Information Engineering, Nanjing University of Aeronautics and Astronautics, Nanjing 210016, China
}

Correspondence should be addressed to Xiangbin Yu, xiyangyang2004@163.com

Received 14 June 2012; Revised 4 August 2012; Accepted 18 August 2012

Academic Editor: Athanasios Panagopoulos

Copyright () 2012 Xiaoyu Dang et al. This is an open access article distributed under the Creative Commons Attribution License, which permits unrestricted use, distribution, and reproduction in any medium, provided the original work is properly cited.

By combining adaptive modulation and automatic repeat request, a cross-layer design (CLD) scheme for MIMO system with antenna selection (AS) and imperfect feedback is presented, and the corresponding performance is studied. Subject to a target packet loss rate and fixed power constraint, the variable switching thresholds of fading gain are derived. According to these results, and using mathematical manipulation, the average spectrum efficiency (SE) and packet error rate (PER) of the system are further derived. As a result, closed-form expressions of the average SE and PER are obtained, respectively. These expressions include the expressions under perfect channel state information as special cases and provide good performance evaluation for the system. Numerical results show that the proposed CLD scheme with antenna selection has higher SE than the existing CLD scheme with space-time block coding, and the CLD scheme with variable switching thresholds outperforms that with conventional-fixed switching thresholds.

\section{Introduction}

With the fast development of modern communication techniques, the demand for high data rate service is growing increasingly in the limited radio spectrum. For this reason, the future wireless communication systems will require spectrally efficient techniques to increase the system capacity. Cross-layer design (CLD), as a good work to improve the spectral efficiency (SE) and system throughput of future wireless systems while meeting the prescribed quality of service (QoS) requirements, has received much attention recently [1-3]. Especially, CLD combining adaptive modulation (AM) and automatic repeat request (ARQ) which is widely accepted as an efficient means to improve the overall performance of transmission in fading channels has been introduced in the improved $3 \mathrm{G}$ scheme, such as high-speed downlink packet access (HSDPA) [4-8]. Antenna selection (AS), which is a one promising approach to achieve the goal of providing performance benefits while significantly decreasing the hardware complexity and cost, has received considerable studies [9-11]. It can provide a good tradeoff between the performance, cost, and complexity and can be realized at both ends. Therefore, effective combination of cross-layer design and antenna selection techniques will receive much attention for practical purpose.

The transmit antenna selection scheme with maximal ratio combining (MRC) is an effective diversity scheme [9, 10]. Based on this antenna selection scheme, the performance analysis of discrete-rate adaptive $M$-ray quadrature amplitude modulation (MQAM) with AS is presented in [12], where the average frame error rate of the system is considered. The performance of adaptive MQAM with antenna selection is studied in $[11,13]$ for perfect and imperfect estimation, respectively, where the average BER and spectrum efficiency are analyzed. By combining adaptive modulation and coding (AMC) and selection of transmit diversity in VBLAST multiplexing multiple-input and multiple-output (MIMO) system, [14] investigates the average throughput of the combined systems through simulations. But the above systems do not consider the superiority of cross-layer design. For this reason, a CLD-combined AMC at the physical layer (PHY) and ARQ protocol at the data link layer (DLL) over 
single-input and single-output fading channel is developed in [4]. Based on the basic idea of CLD in [4], a crosslayer design scheme with space-time block coding (STBC) is presented in Rayleigh fading channel [5]. In terms of different space-time coding schemes, the performance of MIMO system with CLD is studied in [6]. The performance of CLD with STBC is analyzed in optical MIMO system in [7]. Based on perfect channel state information (CSI), a cross-layer approach to transmit antenna selection on the basis of a throughput-based criterion is presented in [3]. Under the feedback constraint, a CLD scheme for multiuser MIMO systems is analyzed in [8].

In all these studies, the system performances of AM with antenna selection or CLD with space-time coding are well analyzed. However, the CLD scheme with antenna selection and the corresponding performance study are not provided in the existing literature. Moreover, the above CLD schemes are basically based on perfect CSI, whereas in practice, the perfect CSI is difficult to obtain due to channel estimation error or feedback delay. Especially, the CLD scheme with variable switching thresholds is not studied, and thus the improvement of system performance is limited. Motivated by the reason above, we will develop a cross-layer design scheme for MIMO system with antenna selection and imperfect feedback information by combining the AM at the PHY and ARQ at the DLL. With ARQ correcting occasional packet errors at the DLL, the stringent error control requirement is alleviated for adaptive modulation at the PHY. Thus, the performance is obviously improved when compared to the usage of AM only at the PHY or incorporation of ARQ only with a fixed modulation. Based on the performance analysis under imperfect CSI, the probability density function (PDF) of effective signal-to-noise ratio (SNR) is presented. Subject to target packet loss rate (PLR) constraint, the variable switching thresholds are derived by using the maximum a posteriori (MAP-) optimal method. According to these results, the closed-form expressions of average packet error rate (PER) and SE of the system are obtained by means of the theoretical analysis and mathematical derivation. With these expressions, the system performance for imperfect CSI can be effectively assessed, and the theoretical expressions for perfect CSI are included. Numerical results show that the proposed CLD with AS has higher SE than the conventional CLD with STBC. Moreover, the derived variable thresholds can reduce the effect of imperfect CSI on the system performance. Namely, the system performance with variable thresholds is superior to that with conventionalfixed thresholds.

The notations throughout this paper are as follows. Bold upper case and lower case letters denote matrices and column vectors, respectively. Some parameters are described as Table 1 for a better understanding.

\section{System Model}

We consider a wireless multiantenna communication system with $N_{t}$ transmit antennas and $N_{r}$ receive antennas operating over a flat and quasistatic Rayleigh fading channel represented by a $N_{r} \times N_{t}$ fading channel matrix $\mathbf{H}=\left\{h_{i n}\right\}$.
TABle 1: Parameter description.

\begin{tabular}{ll}
\hline Parameter Description \\
\hline$N_{t}$ & Number of transmit antenna \\
$\bar{\rho}$ & The average SNR \\
$\mathbf{H}$ & Channel matrix \\
$P_{o}$ & Target PER \\
$N_{r}^{\max }$ & Maximum number of retransmissions \\
$\rho_{k}$ & Fixed switching thresholds (FST) \\
$\overline{\text { PER }}$ & Average PER \\
$\eta_{\text {phy }}$ & Average SE of the system at the PHY \\
$\mathrm{PER}_{k}^{\prime}(\hat{\rho})$ & Estimated instantaneous PER \\
$F_{\mathrm{MAP}}$ & MAP function \\
$\overline{\mathrm{PLR}}$ & Average PLR \\
$c$ & Channel correlation coefficient \\
$J_{0}(\cdot)$ & The zero-order Bessel function of the first kind \\
$N_{r}$ & Number of receive antenna \\
$M_{k}$ & MQAM constellation size \\
$\hat{\mathbf{H}}$ & Time-delayed version of H \\
$P_{\text {loss }}$ & Target PLR \\
$\bar{N}$ & Average number of transmissions per packet \\
$\tilde{\rho}_{k}$ & Variable switching thresholds (VST) \\
$\overline{\mathrm{PER}}$ & Average PER for constellation size $M_{k}$ \\
$\eta$ & Overall average SE of the system \\
$\mathrm{PER}_{k}(\rho)$ & Instantaneous PER \\
$\widetilde{F}_{\mathrm{MAP}}$ & Approximate form of the MAP function \\
$\alpha_{n}$ & Power gains of channel \\
$\sigma_{e}^{2}$ & Estimation error equal to $1-c^{2}$ \\
$I_{v}(\cdot)$ & The $v$ th order-modified Bessel function of the first kind \\
\hline
\end{tabular}

The complex element $h_{\text {in }}$ denotes the channel gain from the $n$th transmit antenna to the $i$ th receive antenna, which is assumed to be constant over a symbol and varied from one symbol to another. The channel gains are modeled as independent complex Gaussian random variables with zero mean and variance 0.5 per real dimension. Let $\alpha_{n}=\sum_{i=1}^{N_{r}}\left|h_{i n}\right|^{2}$ for $n=1, \ldots, N_{t}$ denote the instantaneous channel power gain between the $n$th transmit antenna and all the receive antennas. For the analysis convenience, the power gains $\left\{\alpha_{n}\right\}$ are sorted in ascending order and denoted by $\left\{\beta_{n}\right\}$ such that $\beta_{1} \leq \beta_{2} \leq \cdots \leq \beta_{N_{t}}$. Thus, $\beta_{N_{t}}=\max \left\{\alpha_{n}\right\}$. The basic operation of transmit AS with MRC scheme is to select the antenna corresponding to the maximum $\beta_{N_{t}}$ for transmitting data and use MRC at the receiver for combining the received signals. Thus, the average received SNR after MRC can be expressed as

$$
\rho=\left(\frac{\bar{S}}{\sigma_{n}^{2}}\right) \beta_{N_{t}}=\bar{\rho} \beta_{N_{t}}
$$

where $\bar{S}$ is the average transmitted power; $\sigma_{n}^{2}$ is noise power. $\bar{\rho}=\bar{S} / \sigma_{n}^{2}$ is the average SNR per receive antenna. 
For Rayleigh fading channel, $\alpha_{n}$ is a central $\chi^{2}$-distributed random variable with $2 N_{r}$ degrees of freedom. From equation (2-1-110) in [15], the PDF of $\alpha_{n}$ is given by

$$
f(\alpha)=\alpha^{N_{r}-1} \exp \frac{(-\alpha)}{\Gamma\left(N_{r}\right)}
$$

and the corresponding cumulative distribution function $(\mathrm{CDF})$ is expressed as

$$
F(\alpha)=1-\exp (-\alpha) \sum_{i=0}^{N_{r}-1} \frac{\alpha^{i}}{N_{r} !}
$$

Using the order statistic [16] together with (1) and transformation of random variable, the pdf of $\rho$ can be obtained as follows:

$$
\begin{aligned}
f(\rho)= & \frac{N_{t}}{\bar{\rho} \Gamma\left(N_{r}\right)}\left(\frac{\rho}{\bar{\rho}}\right)^{N_{r}-1} \\
& \times \exp \left(-\frac{\rho}{\bar{\rho}}\right)\left[1-\exp \left(-\frac{\rho}{\bar{\rho}}\right) \sum_{i=0}^{N_{r}-1} \frac{(\rho / \bar{\rho})^{i}}{i !}\right]^{N_{t}-1} \\
= & \frac{N_{t}}{\bar{\rho} \Gamma\left(N_{r}\right)} \sum_{n=0}^{N_{t}-1}(-1)^{n}\left(\begin{array}{c}
N_{t}-1 \\
n
\end{array}\right) \\
& \times \sum_{l=0}^{n\left(N_{r}-1\right)} w_{l}^{n}\left(\frac{\rho}{\bar{\rho}}\right)^{N_{r}-1+l} \exp \left(-\frac{(n+1) \rho}{\bar{\rho}}\right),
\end{aligned}
$$

where $w_{l}^{n}$ is the coefficient of $x^{l}, l=0,1, \ldots, n\left(N_{r}-1\right)$ in the expansion of $\left(\sum_{t=0}^{N_{r}-1}\left(x^{t} / t !\right)\right)^{n}$.

\section{Cross-Layer Design for MIMO Systems with Antenna Selection}

In this section, we will give a cross-layer design scheme for MIMO system with antenna selection, and square MQAM is considered for modulation in the system due to its inherent SE and ease of implementation. For discrete-rate MQAM, the constellation size $M_{k}$ is defined as $\left\{M_{0}=0, M_{1}=\right.$ 2 , and $\left.M_{k}=2^{2 k-2}, k=2, \ldots, K\right\}$, where $M_{0}$ means no data transmission. The instantaneous SNR range is divided into $K$ fading regions with switching thresholds $\left\{\rho_{0}, \rho_{1}, \ldots, \rho_{K}, \rho_{K+1} ; \rho_{0}=0, \rho_{K+1}=+\infty\right\}$. The MQAM of constellation size $M_{k}$ is used for modulation when $\rho$ falls in the $k$ th region $\left[\rho_{k}, \rho_{k+1}\right)$. Hence, the data rate is $d_{k}=$ $\log _{2} M_{k}$ bits/symbol with $d_{0}=0$.

According to [4], the PER of MQAM with twodimensional Gray code over additive white Gaussian noise (AWGN) channel for the received SNR $\rho$ and constellation size $M_{k}$ is approximately given by

$$
\operatorname{PER}_{k}(\rho) \cong \begin{cases}1, & \rho<\rho_{p k}, \\ a_{k} \exp \left(-g_{k} \rho\right), & \rho \geq \rho_{p k},\end{cases}
$$

where $\left\{a_{k}, g_{k}, \rho_{p k}\right\}$ are constellation and packet-size-dependent constants, and they can be obtained by fitting (6) to the exact PER. Specifically, their values can be found in Table 1 in [4].

In our CLD scheme, adaptive modulation at the physical layer and truncated ARQ protocol at the data link layer are employed for cross-layer design. We first define the target packet loss rate for the data link layer as $P_{\text {loss }}$. Since truncated ARQ is used at the data link layer, the packets in error may be retransmitted up to $N_{r}^{\max }$ (maximum number of retransmissions). Hence, the target PER is $P_{o}=P_{\text {loss }}^{1 /\left(N_{r}^{\max }+1\right)}$ at the physical layer, which is generally limited as $P_{o}<1$. The switching thresholds $\left\{\rho_{k}\right\}$ can be set to be the required SNR to achieve the target PER, $P_{o}$, over an AWGN channel. By inverting the $P_{o}$ in (6), we can obtain the switching threshold values as follows:

$$
\rho_{k}= \begin{cases}0 & k=0, \\ \frac{B_{k}}{g_{k}} & k=1, \ldots, K, \\ +\infty & k=K+1,\end{cases}
$$

where $B_{k}=-\ln \left(P_{o} / a_{k}\right)$ is a factor dependent on the choosing of the modulation mode $k$. With the above switching thresholds, the PER and SE performance of MIMO system with cross-layer design and antenna selection may be assessed effectively. Moreover, the system will operate with a PER below target PER $P_{o}$ for perfect CSI when the switching thresholds are chosen according to (7).

\section{Performance Analysis of MIMO System with CLD and AS}

In this section, we will give the performance analysis of CLD with antenna selection (referred to as CLD-AS) under perfect feedback. Closed-form expressions of the average PER and spectrum efficiency are derived, which will be used to assess the performance of CLD-AS for perfect CSI.

Based on the switching thresholds described in (7), and using (5), we can calculate the probability that the SNR, $\rho$, falls in the $k$ th region $\left[\rho_{k}, \rho_{k+1}\right)$, denoted by $P_{k}$, as

$$
\begin{aligned}
P_{k}= & \int_{\rho_{k}}^{\rho_{k+1}} f(\rho) d \rho \\
= & \frac{N_{t}}{\Gamma\left(N_{r}\right)} \sum_{n=0}^{N_{t}-1}(-1)^{n}\left(\begin{array}{c}
N_{t}-1 \\
n
\end{array}\right) \sum_{l=0}^{n\left(N_{r}-1\right)} \frac{w_{l}^{n}}{(n+1)^{N_{r}+l}} \\
& \times\left[\Gamma\left(N_{r}+l, \frac{(n+1) \rho_{k}}{\bar{\rho}}\right)-\Gamma\left(N_{r}+l, \frac{(n+1) \rho_{k+1}}{\bar{\rho}}\right)\right] .
\end{aligned}
$$

For discrete-rate adaptive scheme, the average $\mathrm{SE}$ at the physical layer is defined as the ensemble average of effective transmission rate. So using (5) and the incomplete gamma 
function $\Gamma(\cdot, \cdot)[17]$, the average SE of the system at the PHY with adaptive MQAM and AS can be obtained as

$$
\begin{aligned}
\eta_{\text {phy }}= & \sum_{k=1}^{K} d_{k} \int_{\rho_{k}}^{\rho_{k+1}} f(\rho) d \rho \\
= & \sum_{k=1}^{K} d_{k} P_{k}=\frac{N_{t}}{\Gamma\left(N_{r}\right)} \sum_{k=1}^{K} d_{k} \\
& \times \sum_{n=0}^{N_{t}-1}(-1)^{n}\left(\begin{array}{c}
N_{t}-1 \\
n
\end{array}\right) \sum_{l=0}^{n\left(N_{r}-1\right)} \frac{w_{l}^{n}}{(n+1)^{N_{r}+l}} \\
& \times\left[\Gamma\left(N_{r}+l, \frac{(n+1) \rho_{k}}{\bar{\rho}}\right)-\Gamma\left(N_{r}+l, \frac{(n+1) \rho_{k+1}}{\bar{\rho}}\right)\right] .
\end{aligned}
$$

This equation is a closed-form expression of the average SE of the system at the physical layer.

We define ensemble average PER for MIMO system with CLD and antenna selection as

$$
\begin{aligned}
\overline{\mathrm{PER}} & =\frac{\sum_{k=1}^{K} d_{k} \overline{\mathrm{PER}_{k}}}{\left(\sum_{k=1}^{K} d_{k} \int_{\rho_{k}}^{\rho_{k+1}} f(\rho) d \rho\right)} \\
& =\frac{\sum_{k=1}^{K} d_{k} \overline{\mathrm{PER}_{k}}}{\eta_{\mathrm{phy}}}
\end{aligned}
$$

where $\overline{\mathrm{PER}_{k}}$ denote the average PER for constellation size $M_{k}$. Using (5) and (6), $\overline{\mathrm{PER}_{k}}$ can be obtained as

$$
\begin{aligned}
\overline{\operatorname{PER}}_{k} \cong & \int_{\rho_{k}}^{\rho_{k+1}} \operatorname{PER}_{k}(\rho) f(\rho) d \rho \\
= & \frac{N_{t} a_{k}}{\Gamma\left(N_{r}\right)} \sum_{n=0}^{N_{t}-1}(-1)^{n}\left(\begin{array}{c}
N_{t}-1 \\
n
\end{array}\right) \sum_{l=0}^{n\left(N_{r}-1\right)} \frac{w_{l}^{n}}{\left(\bar{\rho} b_{n, k}\right)^{N_{r}+l}} \\
& \times\left[\Gamma\left(N_{r}+l, b_{n, k} \rho_{k}\right)-\Gamma\left(N_{r}+l, b_{n, k} \rho_{k+1}\right)\right],
\end{aligned}
$$

where $b_{n, k}=g_{k}+(n+1) / \bar{\rho}$. Substituting (11) and (9) into (10), the average PER can be evaluated as follows:

$$
\begin{aligned}
\overline{\mathrm{PER}} \cong & \frac{N_{t}}{\Gamma\left(N_{r}\right) \eta_{\text {phy }}} \sum_{k=1}^{K} d_{k} a_{k} \sum_{n=0}^{N_{t}-1}(-1)^{n} \\
& \times\left(\begin{array}{c}
N_{t}-1 \\
n
\end{array}\right) \sum_{l=0}^{n\left(N_{r}-1\right)} \frac{w_{l}^{n}}{\left(\bar{\rho} b_{n, k}\right)^{N_{r}+l}} \\
& \times\left[\Gamma\left(N_{r}+l, b_{n, k} \rho_{k}\right)-\Gamma\left(N_{r}+l, b_{n, k} \rho_{k+1}\right)\right],
\end{aligned}
$$

where $\eta_{\text {phy }}$ is defined as (9). Equation (12) is a tightly closedform expression of the average PER for MIMO system with CLD and AS. With (12), the average PLR at the data link layer with the maximum number of retransmissions $N_{r}^{\max }$ is

$$
\overline{\mathrm{PLR}}=\overline{\mathrm{PER}}^{N_{r}^{\max }+1}=p_{r}^{N_{r}^{\max }+1},
$$

where $p_{r}=\overline{\mathrm{PER}}$. Thus, the average number of transmissions per packet can be calculated as

$$
\bar{N}=1+p_{r}+p_{r}^{2}+\cdots+p_{r}^{N_{r}^{\max }}=\frac{\left(1-p_{r}^{N_{r}^{\max }+1}\right)}{\left(1-p_{r}\right)}
$$

Using (9) and (14), the overall average SE of the MIMO system with CLD and AS can be attained as

$$
\begin{aligned}
\eta= & \frac{\eta_{\text {phy }}}{\bar{N}} \\
= & \frac{N_{t}}{\bar{N} \Gamma\left(N_{r}\right)} \sum_{k=1}^{K} d_{k} \sum_{n=0}^{N_{t}-1}(-1)^{n}\left(\begin{array}{c}
N_{t}-1 \\
n
\end{array}\right) \\
& \times \sum_{l=0}^{n\left(N_{r}-1\right)} \frac{w_{l}^{n}}{(n+1)^{N_{r}+l}} \\
& \times\left[\Gamma\left(N_{r}+l, \frac{(n+1) \rho_{k}}{\bar{\rho}}\right)-\Gamma\left(N_{r}+l, \frac{(n+1) \rho_{k+1}}{\bar{\rho}}\right)\right] .
\end{aligned}
$$

When $N_{r}^{\max }$ is set to be zero, then $\bar{N}=1$, and (15) is reduced to $\eta_{\text {phy }}$, which corresponds to the average SE for AM at the physical layer only.

\section{Performance of CLD with Antenna Selection under Imperfect Feedback}

5.1. Variable Switching Thresholds. In the above section, we analyze the performance of CLD-AS scheme assuming the CSI is perfectly available at the receiver and the transmitter. In practice, the channel state information is imperfect due to imperfect feedback. So in this section, we investigate the effect of imperfect feedback (delayed feedback) on the performance of CLD-AS system. The channel is assumed to be perfectly known at the receiver and is fed back to the transmitter with time delay $\tau$. $\hat{\mathbf{H}}$ is the $\tau$ time-delayed version of $\mathbf{H}$, and it is drawn from the same Gaussian process as $\mathbf{H}$. The entries of $\hat{\mathbf{H}},\left\{\hat{h}_{i n}\right\}$ are correlated with $\left\{h_{i n}\right\}$ with correlation coefficient $c=J_{0}\left(2 \pi f_{d} \tau\right)$ [18], where $J_{0}(\cdot)$ is the zero-order Bessel function of the first kind [17], and $f_{d}$ is the maximum Doppler frequency [15]. At the transmitter, the transmitter will use the delayed CSI, $\hat{\rho}=\bar{\rho} \widehat{\beta}_{N_{t}}$, to adjust the modulation mode. According to the above analysis, $\hat{\rho}$ will have the same PDF as the perfect CSI $\rho$. Thus, the PDF of $\hat{\rho}$ can be obtained as

$$
\begin{aligned}
f(\hat{\rho})= & \frac{N_{t}}{\bar{\rho} \Gamma\left(N_{r}\right)} \sum_{n=0}^{N_{t}-1}(-1)^{n}\left(\begin{array}{c}
N_{t}-1 \\
n
\end{array}\right) \\
& \times \sum_{l=0}^{n\left(N_{r}-1\right)} w_{l}^{n}\left(\frac{\hat{\rho}}{\bar{\rho}}\right)^{N_{r}-1+l} \exp \left(-\frac{(n+1) \hat{\rho}}{\bar{\rho}}\right) .
\end{aligned}
$$


For delayed feedback, according to $[18,19]$, the conditional PDF of $\rho$ given $\hat{\rho}$ is expressed as

$$
\begin{aligned}
f(\rho \mid \hat{\rho})= & \frac{1}{\sigma_{e}^{2} \bar{\rho}}\left(\frac{\rho}{c^{2} \hat{\rho}}\right)^{\left(N_{r}-1\right) / 2} I_{N_{r}-1}\left(\frac{2 c \sqrt{\rho \hat{\rho}}}{\bar{\rho} \sigma_{e}^{2}}\right) \\
& \times \exp \left(-\frac{\left(\rho+c^{2} \hat{\rho}\right)}{\bar{\rho} \sigma_{e}^{2}}\right),
\end{aligned}
$$

where $\sigma_{e}^{2}=1-c^{2}$, and $I_{v}(\cdot)$ is the $v$ th order-modified Bessel function of the first kind [17]. With (16) and (17), the joint pdf of $\rho$ and $\hat{\rho}$ can be obtained as

$$
\begin{aligned}
f_{\rho, \hat{\rho}}(\rho, \hat{\rho})= & \frac{N_{t}}{\bar{\rho} \Gamma\left(N_{r}\right)} \sum_{n=0}^{N_{t}-1}(-1)^{n} \\
& \times\left(\begin{array}{c}
N_{t}-1 \\
n
\end{array}\right) \sum_{l=0}^{n\left(N_{r}-1\right)} w_{l}^{n}\left(\frac{\hat{\rho}}{\bar{\rho}}\right)^{N_{r}-1+l} \\
& \times \exp \left(-\frac{(n+1) \hat{\rho}}{\bar{\rho}}\right) \frac{1}{\sigma_{e}^{2} \bar{\rho}}\left(\frac{\rho}{c^{2} \hat{\rho}}\right)^{\left(N_{r}-1\right) / 2} \\
& \times I_{N_{r}-1}\left(\frac{2 c \sqrt{\rho \hat{\rho}}}{\bar{\rho} \sigma_{e}^{2}}\right) \exp \left(-\frac{\left(\rho+c^{2} \hat{\rho}\right)}{\bar{\rho} \sigma_{e}^{2}}\right) .
\end{aligned}
$$

Considering that the switching thresholds in (7) are fixed, they can not adapt to the change of delayed feedback information. Thus, the performance loss will happen when the feedback has a delay. For this, we will present a variable threshold method for imperfect feedback by employing the Bayes' theorem [20] and MAP criterion [21, 22]. With the variable thresholds, the system performance will be effectively improved and outperforms that with fixed thresholds (7).

In order to develop the threshold adaptation algorithm for switching thresholds, the maximum a posterior-based PER estimation will be adopted. Namely, the estimated instantaneous PER, $\operatorname{PER}_{k}^{\prime}(\hat{\rho})$, will be used to derive the variable thresholds. $\operatorname{PER}_{k}^{\prime}(\hat{\rho})$ can be obtained by maximizing the following conditional PDF:

$$
f_{\mathrm{PER}_{k} \mid \hat{\rho}}\left(\mathrm{PER}_{k} \mid \hat{\rho}\right)=f_{\mathrm{PER}_{k}, \hat{\rho}} \frac{\left(\mathrm{PER}_{k}, \hat{\rho}\right)}{f(\hat{\rho})} .
$$

According to the functional relationship between $\operatorname{PER}_{k}(\rho)$ and $\rho$ in (6), using the analysis method in [21], the joint PDF of $\operatorname{PER}_{k}(\rho)$ and $\hat{\rho}, f_{\operatorname{PER}_{k}, \hat{\rho}}\left(\operatorname{PER}_{k}, \hat{\rho}\right)$, is derived as

$$
\begin{aligned}
f_{\mathrm{PER}_{k}, \hat{\rho}}\left(\mathrm{PER}_{k}, \hat{\rho}\right)= & \left|\frac{\partial \rho}{\partial \operatorname{PER}_{k}}\right| f_{\rho, \hat{\rho}}\left(\operatorname{PER}_{k}^{-1}(\rho), \hat{\rho}\right) \\
= & \frac{A}{g_{k} a_{k}}\left(\frac{\xi^{2}}{g_{k}}\right)^{\left(N_{r}-1\right) / 2} I_{N_{r}-1}(\xi \mu \sqrt{\hat{\rho}}) \\
& \times \exp \left(\xi^{2}-\frac{\xi^{2}}{\left(g_{k} \bar{\rho} \sigma_{e}^{2}\right)}\right),
\end{aligned}
$$

where $\xi=\sqrt{\ln \left(a_{k} / \mathrm{PER}_{k}\right)}, \operatorname{PER}_{k}^{-1}(\rho)$ is the inverse function of $\operatorname{PER}_{k}(\rho)$, and thus we have $\rho=\operatorname{PER}_{k}^{-1}(\rho)=\xi^{2} / g_{k} \cdot \mu_{k}=$ $2 c \sqrt{g_{k}} / v_{k}, v_{k}=g_{k} \bar{\rho} \sigma_{e}^{2}$, and $A$ is written as

$$
\begin{aligned}
A= & \frac{N_{t} c^{1-N_{r}}}{\sigma_{e}^{2} \Gamma\left(N_{r}\right)} \sum_{n=0}^{N_{t}-1}(-1)^{n}\left(\begin{array}{c}
N_{t}-1 \\
n
\end{array}\right) \\
& \times \sum_{l=0}^{n\left(N_{r}-1\right)} w_{l}^{n} \frac{\hat{\rho}^{1.5\left(N_{r}-1\right)+l}}{\bar{\rho}^{N_{r}+1+l}} \exp \left(-\left(\frac{n+1}{\bar{\rho}}+\frac{c^{2}}{\sigma_{e}^{2} \bar{\rho}}\right) \hat{\rho}\right) .
\end{aligned}
$$

According to (20) and omitting the terms not related to $\xi$, we may define the following MAP function as

$$
F_{\mathrm{MAP}}=\xi^{N_{r}-1} I_{N_{r}-1}(\xi \mu) \exp \left(\xi^{2}-\frac{\xi^{2}}{v_{k}}\right) .
$$

Using the approximate expression $I_{n}(z) \approx \exp (z) / \sqrt{2 \pi z}$ for large $z$ [23], (22) can be approximated as

$$
\widetilde{F}_{\mathrm{MAP}}=\frac{\xi^{N_{r}-1.5}}{(\sqrt{2 \pi \mu \sqrt{\hat{\rho}}}) \exp \left(\xi \mu \sqrt{\hat{\rho}}+\xi^{2}-\xi^{2} / \nu_{k}\right)},
$$

where $\widetilde{F}_{\text {MAP }}$ denotes the approximate form of the MAP function $F_{\text {MAP }}$ for large SNR $\hat{\rho}$. Based on (23), by solving $\partial \widetilde{F}_{\mathrm{MAP}} / \partial \mathrm{PER}_{k}=\left(\partial \widetilde{F}_{\mathrm{MAP}} / \partial \xi\right) \cdot\left(\partial \xi / \partial \mathrm{PER}_{k}\right)=0$, we can obtain the approximated expression of the estimated PER as

$$
\operatorname{PER}_{k}^{\prime}(\hat{\rho}) \cong a_{k} \exp \left(\frac{-c^{2} g_{k} \hat{\rho}}{\left(g_{k} \sigma_{e}^{2}-1\right)^{2}}\right) .
$$

This estimated PER includes imperfect CSI, such as channel correlation coefficient related to the time delay, and thus it can be used to obtain the switching thresholds adaptation to feedback delay. Let $\operatorname{PER}_{k}^{\prime}(\hat{\rho})$ be target PER $P_{0}$, then with (24), we can obtain the variable thresholds dependent on the feedback delay as

$$
\begin{aligned}
\tilde{\rho}_{k} & \cong\left[\frac{-\ln \left(P_{0} / a_{k}\right)}{g_{k}}\right] \frac{\left(g_{k} \sigma_{e}^{2}-1\right)^{2}}{c^{2}} \\
& =\frac{\left(B_{k} / g_{k}\right)\left(g_{k} \sigma_{e}^{2}-1\right)^{2}}{c^{2}} .
\end{aligned}
$$

Unlike the fixed switching thresholds in (7) (which do not consider the impact of delayed feedback at the transmitter), the variable switching thresholds in (25) take the effect of delayed feedback into account when performing rate adaptation at the transm+itter. Thus, with (25), the impact of feedback delay may be reduced, and reliable PER performance will be obtained. When the feedback is perfect (i.e., no delay), $c^{2}=1,(25)$ is reduced to (7), and so the variable switching thresholds include the fixed switching thresholds as special cases.

5.2. Performance Analysis. In this subsection, we will use the derived variable thresholds for performance analysis of the system. With (16) and (25), we can obtain the average SE 
of the MIMO system with adaptive MQAM and antenna selection at the PHY as

$$
\begin{aligned}
\eta_{\text {phy }}= & \frac{N_{t}}{\Gamma\left(N_{r}\right)} \sum_{k=1}^{K} d_{k} \sum_{n=0}^{N_{t}-1}(-1)^{n} \\
& \times\left(\begin{array}{c}
N_{t}-1 \\
n
\end{array}\right) \sum_{l=0}^{n\left(N_{r}-1\right)} \frac{w_{l}^{n}}{(n+1)^{N_{r}+l}} \\
& \times\left[\Gamma\left(N_{r}+l, \frac{(n+1) \tilde{\rho}_{k}}{\bar{\rho}}\right)-\Gamma\left(N_{r}+l, \frac{(n+1) \tilde{\rho}_{k+1}}{\bar{\rho}}\right)\right] .
\end{aligned}
$$

Equation (26) is a closed-form expression of average SE of the system with imperfect CSI at the PHY. Using this expression, we can evaluate the average SE at the PHY without ARQ protocol in the presence of imperfect CSI. With (16), (17), (25), and (26), the average PER of the CLD-AS with imperfect CSI can be expressed as

$$
\begin{aligned}
\overline{\mathrm{PER}}= & \left(\frac{1}{\eta_{\text {phy }}}\right) \sum_{k=1}^{K} d_{k} \int_{\tilde{\rho}_{k}}^{\tilde{\rho}_{k+1}} \int_{0}^{\infty} \operatorname{PER}_{\rho \mid \hat{\rho}} f(\rho \mid \hat{\rho}) d \rho f(\hat{\rho}) d \hat{\rho} \\
\cong & \left(\frac{1}{\eta_{\text {phy }}}\right) \sum_{k=1}^{K} d_{k} \int_{\tilde{\rho}_{k}}^{\tilde{\rho}_{k+1}} \int_{0}^{\infty} a_{k} \\
& \times \exp \left(-g_{k} \rho\right) f(\rho \mid \hat{\rho}) d \rho f(\hat{\rho}) d \hat{\rho} .
\end{aligned}
$$

Substituting (16) and (17) into (27), and solving the double integrals, we have

$$
\begin{aligned}
\overline{\mathrm{PER}} \cong & \frac{N_{t}}{\Gamma\left(N_{r}\right) \eta_{\text {phy }}} \sum_{k=1}^{K} \frac{d_{k} a_{k}}{\left(1+g_{k}\left(1-c^{2}\right) \bar{\rho}\right)^{M}} \sum_{n=0}^{N_{t}-1}(-1)^{n} \\
& \times\left(\begin{array}{c}
N_{t}-1 \\
n
\end{array}\right) \sum_{l=0}^{n\left(N_{r}-1\right)} \frac{w_{l}^{n}}{\left(\bar{\rho} \hat{b}_{n, k}\right)^{N_{r}+l}} \\
& \times\left[\Gamma\left(N_{r}+l, \hat{b}_{n, k} \tilde{\rho}_{k}\right)-\Gamma\left(N_{r}+l, \hat{b}_{n, k} \tilde{\rho}_{k+1}\right)\right]
\end{aligned}
$$

where $\hat{b}_{n, k}=(n+1) / \bar{\rho}+c^{2} g_{k} /\left[1+g_{k} \bar{\rho}\left(1-c^{2}\right)\right]$. The above equation is a closed-form average PER expression of CLDAS with imperfect feedback.

Following the derivation approaches under perfect CSI, the overall average SE of CLD-AS can be obtained by using (26) and (28), that is,

$$
\begin{aligned}
\eta= & \frac{\eta_{\text {phy }}}{\bar{N}} \\
= & \frac{N_{t}}{\bar{N} \Gamma\left(N_{r}\right)} \sum_{k=1}^{K} d_{k} \sum_{n=0}^{N_{t}-1}(-1)^{n}\left(\begin{array}{c}
N_{t}-1 \\
n
\end{array}\right) \\
& \times \sum_{l=0}^{n\left(N_{r}-1\right)} \frac{w_{l}^{n}}{(n+1)^{N_{r}+l}} \\
& \times\left[\Gamma\left(N_{r}+l, \frac{(n+1) \tilde{\rho}_{k}}{\bar{\rho}}\right)-\Gamma\left(N_{r}+l, \frac{(n+1) \tilde{\rho}_{k+1}}{\bar{\rho}}\right)\right],
\end{aligned}
$$

where $\bar{N}=\left(1-\overline{\mathrm{PER}}^{N_{r}^{\max }+1}\right) /(1-\overline{\mathrm{PER}})$, and $\overline{\mathrm{PER}}$ is from (28). When the feedback has no delay, $c^{2}=1$. Using $c^{2}=1$

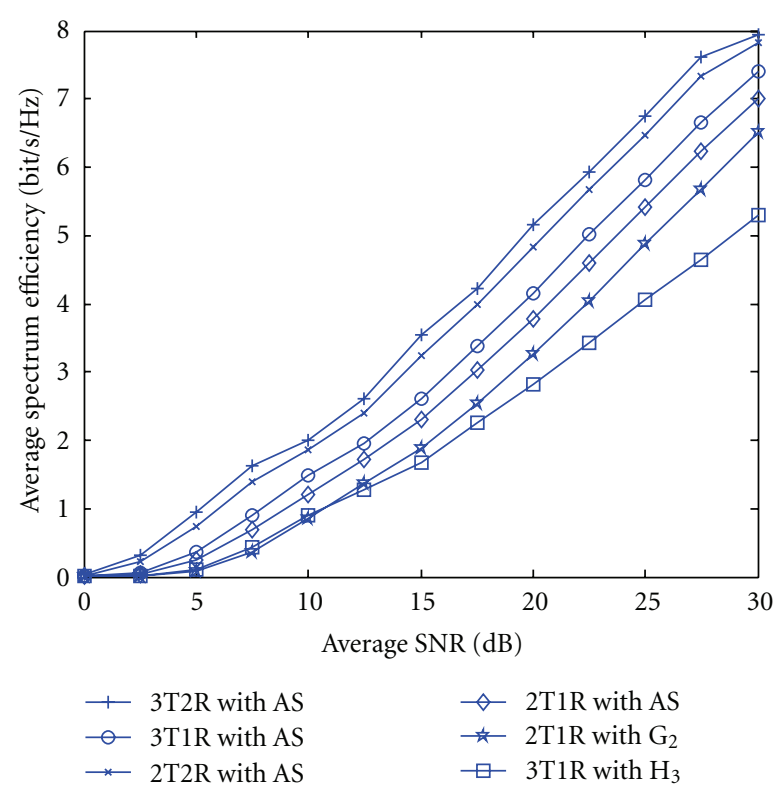

Figure 1: Average SE of CLD-AS with different transmit antennas and one receive antenna.

in (28) and (29), (28) and (29) will be reduced to (12) and (15) under perfect feedback, respectively. Hence, the derived SE and PER expressions under imperfect feedback include those under perfect feedback as special cases. With (28) and (29), we can assess the impact of time delay on the PER and SE performance of the CLD-AS. When the correlation coefficient $c$ decreases due to the impact of feedback delay, the variable switching thresholds will increase according to (25). Correspondingly, the PER performance is improved, which will bring about the decrease of the average number of transmission $\bar{N}$. Thus, the overall average SE is effectively enhanced in terms of (29) when $\eta_{\text {phy }}$ is fixed. Based on this, the CLD scheme with the variable thresholds will be superior to that with the fixed thresholds.

\section{Numerical Results and Theoretical Evaluation}

In this section, we will use the derived performance formulae to evaluate the average SE and PER performance of the MIMO system with CLD and AS over Rayleigh fading channel. The channel is assumed to be quasistatic flat fading and Gray code is employed to map the data bits to MQAM constellations. The set of MQAM constellations is $\left\{M_{k}\right\}_{k=0,1, \ldots, 5}=\{0,2,4,16,64,256\}$. The target packet loss rate in the data link layer is $P_{\text {loss }}=10^{-3}$, and the maximum number of ARQ retransmissions is $N_{r}^{\max }=2$. Thus, the target PER, $P_{o}$, is equal to 0.1 . In the following figures, $x \mathrm{~T} y \mathrm{R}$ denotes a multiple-antenna system with $x$ transmit antennas and $y$ receive antennas.

In Figure 1, we plot the average spectrum efficiency of the CLD-AS for different transmit antennas and different receive antennas under perfect CSI. The CLD with STBC (CLD-STBC) system in [5] is used for comparison, where 


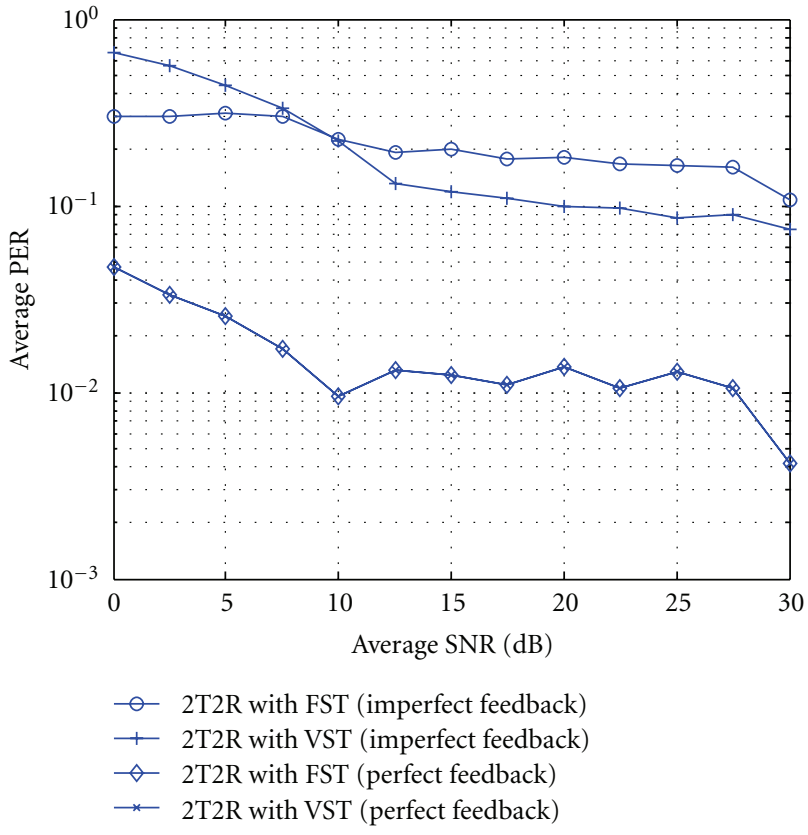

Figure 2: Average PER of CLD-AS with two transmit antennas and two receive antennas.

$\mathrm{G}_{2}, \mathrm{H}_{3}$ codes are employed for STBC schemes. The average SE is calculated by using (15) with the switching thresholds defined in (7). As shown in Figure 1, CLD is able to increase SE with SNR. For the same number of receive antennas, the SE of CLD with three transmit antennas is higher than that with two transmit antennas because the former has greater diversity than the latter; that is, $3 \mathrm{~T} 1 \mathrm{R}$ and $3 \mathrm{~T} 2 \mathrm{R}$ systems outperform the corresponding $2 \mathrm{~T} 1 \mathrm{R}$ and $2 \mathrm{~T} 2 \mathrm{R}$ systems, respectively. Due to the same reason, the SE of CLD with two receive antennas is higher than that with single receive antenna for the same number of transmit antennas. Moreover, we can see that the SE of CLD-AS is much higher than CLD-STBC. It is because the proposed CLD-AS can make full use of the antenna with maximal channel power gain to transmit data so that the received signal power at the receiver is maximized. Especially when transmit antenna is larger than two, the SE superiority of the proposed CLD scheme becomes more significant because the rate of STBC is less than 1 in the case of more than two transmit antennas. Hence, the SE of CLD-AS with 3 transmit antennas is much higher than that of CLD-STBC with $\mathrm{H}_{3}$ (3/4-rate). The above results show that the derived formulae are valid.

In Figure 2, we plot the average PER of the CLD-AS system with two transmit antennas and two receive antennas, where perfect feedback (zero-delay feedback, i.e., $f_{d} \tau=0$ ) and imperfect feedback (i.e., $f_{d} \tau=0.1$ ) are considered. The fixed switching thresholds (FST) defined in (7) and variable switching thresholds (VST) defined in (25) are used for comparison. The average PER is calculated by (12) for perfect feedback and (28) for imperfect feedback. It is shown in Figure 2 that the average PER values are below the target PER (i.e., $P_{o}$ ) for perfect feedback, which means that the CLD is successful in increasing SE while the target PER is

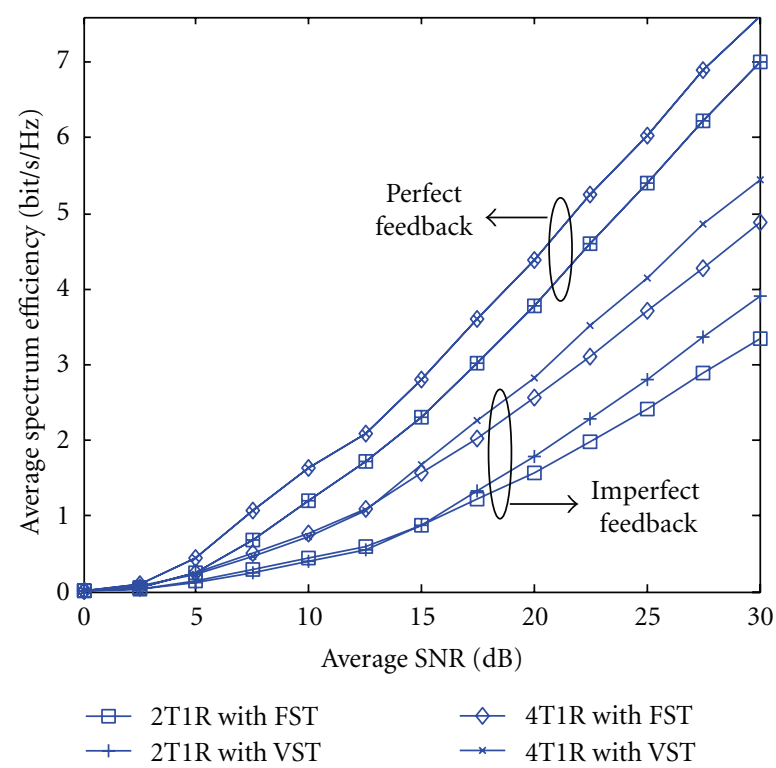

FIGURE 3: Average SE of CLD-AS with different transmit antennas and one receive antenna.

maintained. When the feedback is imperfect, however, the PER performance degrades due to the impact of feedback delay, and the resultant PER starts to fail to meet the target PER, especially for FST method. Besides, the system PER performance with FST is worse than that with VST. This is because the latter can adapt to the delayed feedback. Thus, it can utilize the feedback information to reduce the effect of feedback delay on the performance. Whereas for the former, it is fixed for different feedback, and thus the impact of feedback delay is difficult to overcome. Based on this, the presented VST method is more effective than the FST method against the feedback delay, especially at high SNR. This is because the proposed VST method is obtained based on the approximation under high SNR. Namely, the approximate expression of the modified Bessel function of the first kind $I_{n}(x) \approx \exp (x) / \sqrt{2 \pi x}$ is used for deriving the VST method, and this approximation is much more effective for very large $x$. When $x$ is small, however, this approximation is not feasible; that is, it can not reflect the actual values of $I_{n}(x)$. Thus, our VST scheme is suitable for high SNR, while for low SNR, it will no longer be valid for adaptation to the delayed feedback. When feedback is perfect, the VST is identical to the FST, and correspondingly, their PER curves are the same.

In Figure 3, we plot the average SE of the CLD-AS system with different transmit antennas and one receive antenna for perfect feedback $\left(f_{d} \tau=0\right)$ and imperfect feedback $\left(f_{d} \tau=0.1\right)$, where the FST and VST methods are used for performance evaluation. The average SE is calculated by (15) and (29) for perfect and imperfect cases, respectively. From Figure 3, we can see that the CLD scheme using 4T1R performs better than that using 2T1R because the former has greater diversity than the latter. Due to the effect of feedback delay, the system with imperfect CSI is worse than that with 


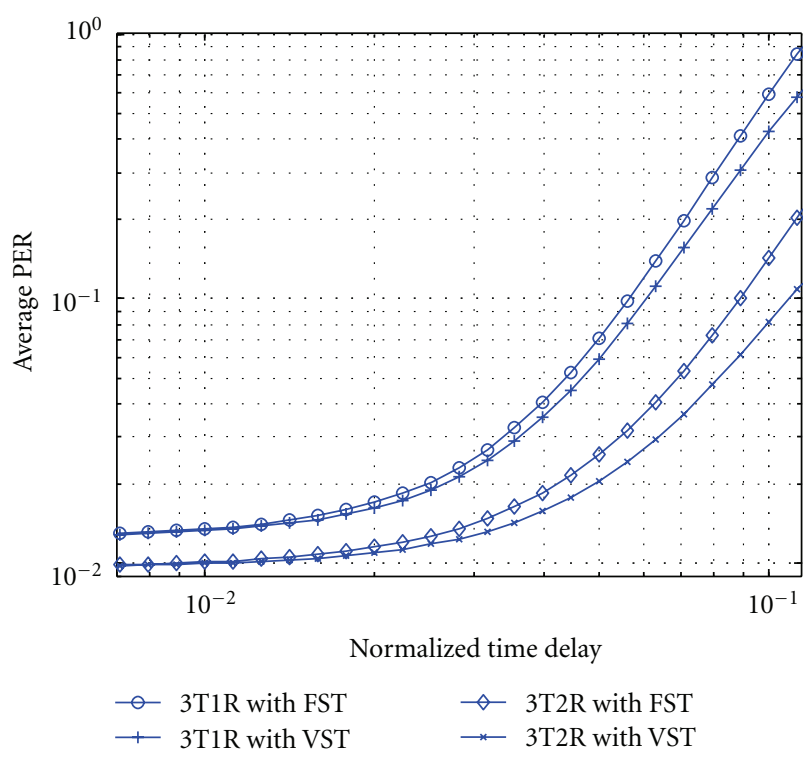

FIGURE 4: Effect of normalized time delay on average PER of CLDAS with three transmit antennas and different receive antennas.

perfect CSI. Besides, the SE with VST is obviously higher than that with FST, especially at high SNR. This is because the application of VST can lower the system PER (as shown in Figure 3), which will bring about the decrease of the average number of transmissions $\bar{N}$ according to (14). As a result, the increase of overall average SE is obtained. For perfect feedback (where VST is the same as FST), the average SE of the system using VST and FST will be identical. The above results further verify the effectiveness of the presented VST method.

We plot the average PER versus normalized time delay $\left(f_{d} \tau\right)$ in Figure 4 for the CLD-AS with three transmit antennas and different receive antennas. The average SNR is set equal to $22 \mathrm{~dB}$. The results show that the CLD-AS can tolerate the normalized time delay up to about 0.01 with a slight degradation in the average PER. But when $f_{d} \tau$ increases beyond 0.01 , the PER performance will degrade increasingly. The 3T1R system fails to meet the target PER $\left(P_{0}=0.1\right)$ at $f_{d} \tau=0.056$ for FST and at $f_{d} \tau=0.062$ for VST, while the $3 \mathrm{~T} 2 \mathrm{R}$ system fails at $f_{d} \tau=0.088$ for FST and at $f_{d} \tau=$ 0.107 for VST. From these results, we can see that the system with the presented VST method can tolerate higher time delay than the conventional FST method. This is because the derived VST method is obtained based on the delayed feedback information, and correspondingly, the impact of delayed feedback on performance is relatively small, while the latter does not consider the delayed information. As a result, the former is superior to the latter. When the time delay is smaller $(<0.01)$, however, their performance is almost the same. Besides, it is found that the PER performance of the 3T1R system is more sensitive to time delay than the 3T2R system. This is due to the fact that $3 \mathrm{~T} 2 \mathrm{R}$ system employs multiple receive antennas and has greater diversity than $3 \mathrm{~T} 1 \mathrm{R}$ system.

\section{Conclusions}

We have studied the performance of cross-layer design with antenna selection for both perfect and imperfect CSIs over Rayleigh fading channel. Subject to the target PER constraint and delayed feedback, we have derived the variable switching thresholds by means of the maximum a posteriori method and approximate PER expression. As a result, closedform variable thresholds are obtained, and they include the conventional-fixed thresholds as special cases. Based on the obtained switching thresholds, the closed-form expressions of the average PER and SE are derived for both perfect and imperfect CSIs. Using these expressions, the performance of CLD-AS system is effectively assessed. The impact of delayed CSI on the system performance is analyzed, and the analysis shows that CLD-AS systems are insensitive to the time delay $<0.01$. Moreover, the presented variable thresholds method can adapt to the delayed feedback information and reduce the effect of feedback delay. Thus, the system performance is superior to that with the conventional-fixed thresholds. Numerical results show the presented CLD with AS can obtain higher SE than the existing CLD with space-time coding the PER and SE performance of the system with variable thresholds both perform better than those of the system with fixed thresholds, especially for large time delay and/or large SNR.

\section{Appendix}

In this appendix, some parameters are provided in Table 1 for understanding the derived equations easily.

\section{Acknowledgments}

The authors would like to thank the anonymous reviewer and the editor for their valuable comments. This work was supported in part by National Natural Science Foundation of China (61172078, 61172077), Doctoral Fund of Ministry of Education of China (20093218120021), and the Fundamental Research Funds for the Central Universities (NS2012075).

\section{References}

[1] J. Ramis and G. Femenias, "Cross-layer design of adaptive multirate wireless networks using truncated HARQ," IEEE Transactions on Vehicular Technology, vol. 60, no. 3, pp. 944954, 2011.

[2] R. Cohen, G. Grebla, and L. Katzir, "Cross-layer hybrid FEC/ ARQ reliable multicast with adaptive modulation and coding in broadband wireless networks," IEEE/ACM Transactions on Networking, vol. 18, pp. 1908-1920, 2010.

[3] J. L. Vicario, M. A. Lagunas, and C. Antón-Haro, "A cross-layer approach to transmit antenna selection," IEEE Transactions on Wireless Communications, vol. 5, no. 8, pp. 1993-1997, 2006.

[4] Q. Liu, S. Zhou, and G. B. Giannakis, "Cross-layer combining of adaptive modulation and coding with truncated ARQ over wireless links," IEEE Transactions on Wireless Communications, vol. 3, no. 5, pp. 1746-1755, 2004.

[5] A. Maaref and S. Aïssa, "A cross-layer design for mimo rayleigh fading channels," in Proceedings of the Canadian Conference on 
Electrical and Computer Engineering (CCECE '04), pp. 22472250, May 2004.

[6] X. F. Lu, G. X. Zhu, G. Liu, and L. Li, "A cross-layer design over MIMO rayleigh fading channels," in Proceedings of the International Conference on Wireless Communications, Networking and Mobile Computing (WCNM '05), pp. 30-33, September 2005.

[7] S. A. R. Zaidi and M. Hafeez, "Cross layer design for orthogonal space time block coded optical MIMO systems," in Proceedings of the 5th IEEE and IFIP International Conference on Wireless and Optical Communications Networks (WOCN '08), pp. 1-5, May 2008.

[8] J. Qi and S. Aïssa, "Cross-Layer design for multiuser MIMO MRC systems with feedback constraints," IEEE Transactions on Vehicular Technology, vol. 58, no. 7, pp. 3347-3360, 2009.

[9] A. F. Molisch, M. Z. Win, Y. S. Choi, and J. H. Winters, "Capacity of MIMO systems with antenna selection," IEEE Transactions on Wireless Communications, vol. 4, no. 4, pp. 1759-1771, 2005.

[10] Z. Chen, J. Yuan, and B. Vucetic, "Analysis of transmit antenna selection/maximal-ratio combining in Rayleigh fading channels," IEEE Transactions on Vehicular Technology, vol. 54, no. 4, pp. 1312-1321, 2005.

[11] X. B. Yu, S. H. Leung, and W. K. Wong, "Performance analysis of variable-power adaptive modulation with antenna selection over Rayleigh fading channels," IET Communications, vol. 5, no. 10, pp. 1394-1404, 2011.

[12] J. Kim, I. Kim, S. Ro, D. Hong, and C. Kang, "Performance analysis of adaptive modulation system combined with transmit/receive diversity," in Proceedings of the Vehicular Technology Conference, pp. 837-841, May 2002.

[13] X. Zhu and D. Yuan, "Performance analysis of adaptive modulation in MIMO system using transmit antenna selection with alamouti scheme," in Proceedings of the International Conference on Wireless Communications, Networking and Mobile Computing (WiCOM '08), pp. 1-4, October 2008.

[14] S. Ro, I. Hwang, D. Hong, C. Kang, and M. Kang, "Adaptive modulation and coding of MIMO in next generation mobile systems," in Proceedings of International Conference Computational Science and Its Applications (ICCSA '03), vol. 2668, pp. 445-454, 2003.

[15] J. G. Proakis, Digital Communications, McGraw-Hill, New York, NY, USA, 5th edition, 2007.

[16] H. A. David, Order Statistics, Wiley, New York, NY, USA, 2nd edition, 1981.

[17] I. S. Gradshteyn and I. M. Ryzhik, Table of Integrals, Series, and Products, Academic, San Diego, Calif, USA, 7th edition, 2007.

[18] M. S. Alouini and A. J. Goldsmith, "Adaptive modulation over Nakagami fading channels," Wireless Personal Communications, vol. 13, no. 1, pp. 119-143, 2000.

[19] Y. Ko and and C. Tepedelenlioglu, "Orthogonal space-time block coded rate-adaptive modulation with outdated feedback," IEEE Transactions on Wireless Communications, vol. 5, pp. 290-295, 2006.

[20] A. Papoulis and and S. U. Pillai, Probability, Random Variables and Stochastic Processes, McGraw Hill, New York, NY, USA, 4th edition, 2002.

[21] L. T. Ong, M. Shikh-Bahaei, and J. A. Chambers, "Variable rate and variable power MQAM system based on Bayesian bit error rate and channel estimation techniques," IEEE Transactions on Communications, vol. 56, no. 2, pp. 177-182, 2008.

[22] S. D. Lee and Y. C. Ko, "Joint variable threshold M-QAM and ARQ for Nakagami-m fading channel with feedback delay,"
IEEE Communications Letters, vol. 14, no. 2, pp. 139-141, 2010.

[23] M. Abramowitz and and I. A. Stegun, Handbook of Mathematical Functions with Formulas, Graphs, and Mathematical Tables, Dover, New York, NY, USA, 1974. 

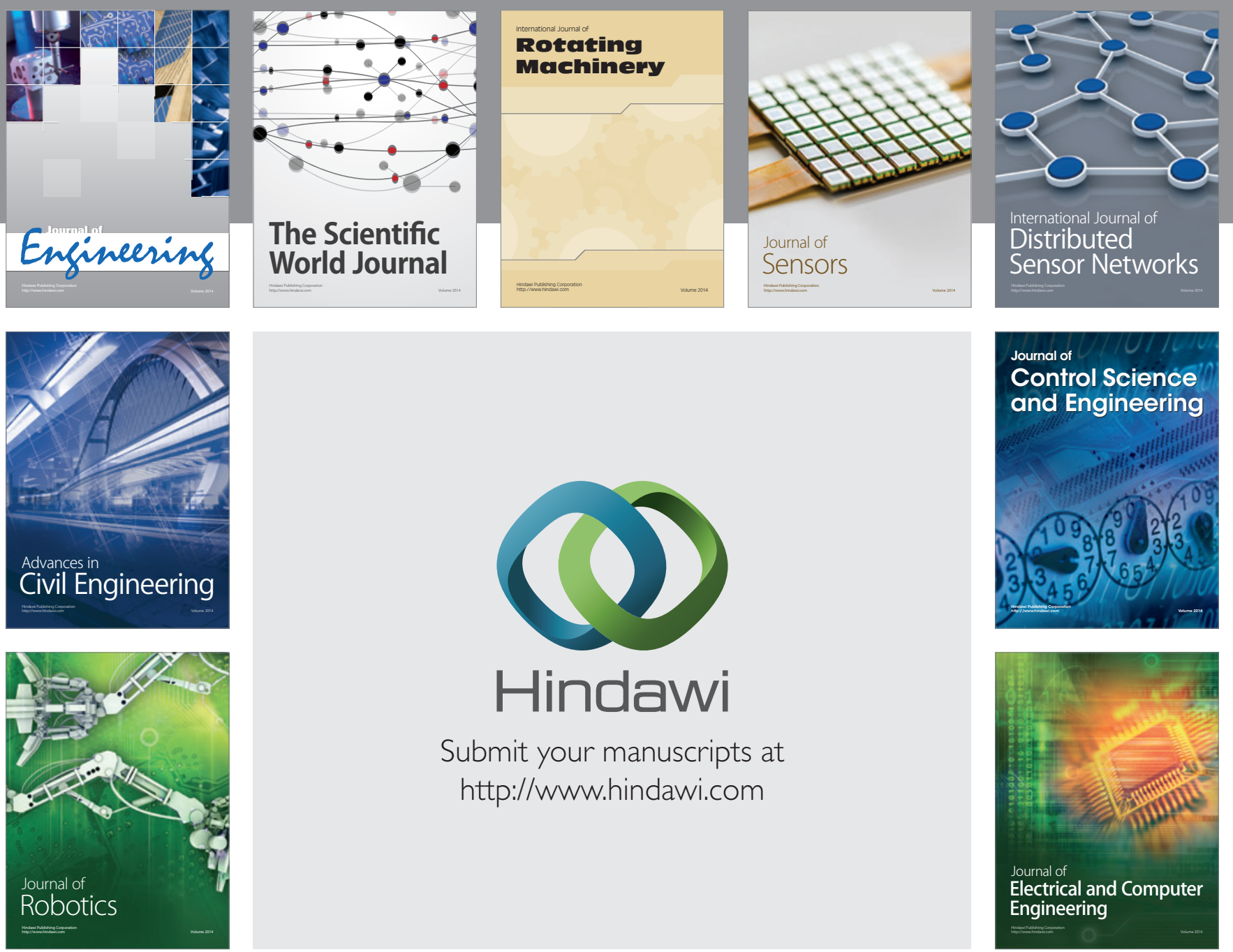

Submit your manuscripts at

http://www.hindawi.com
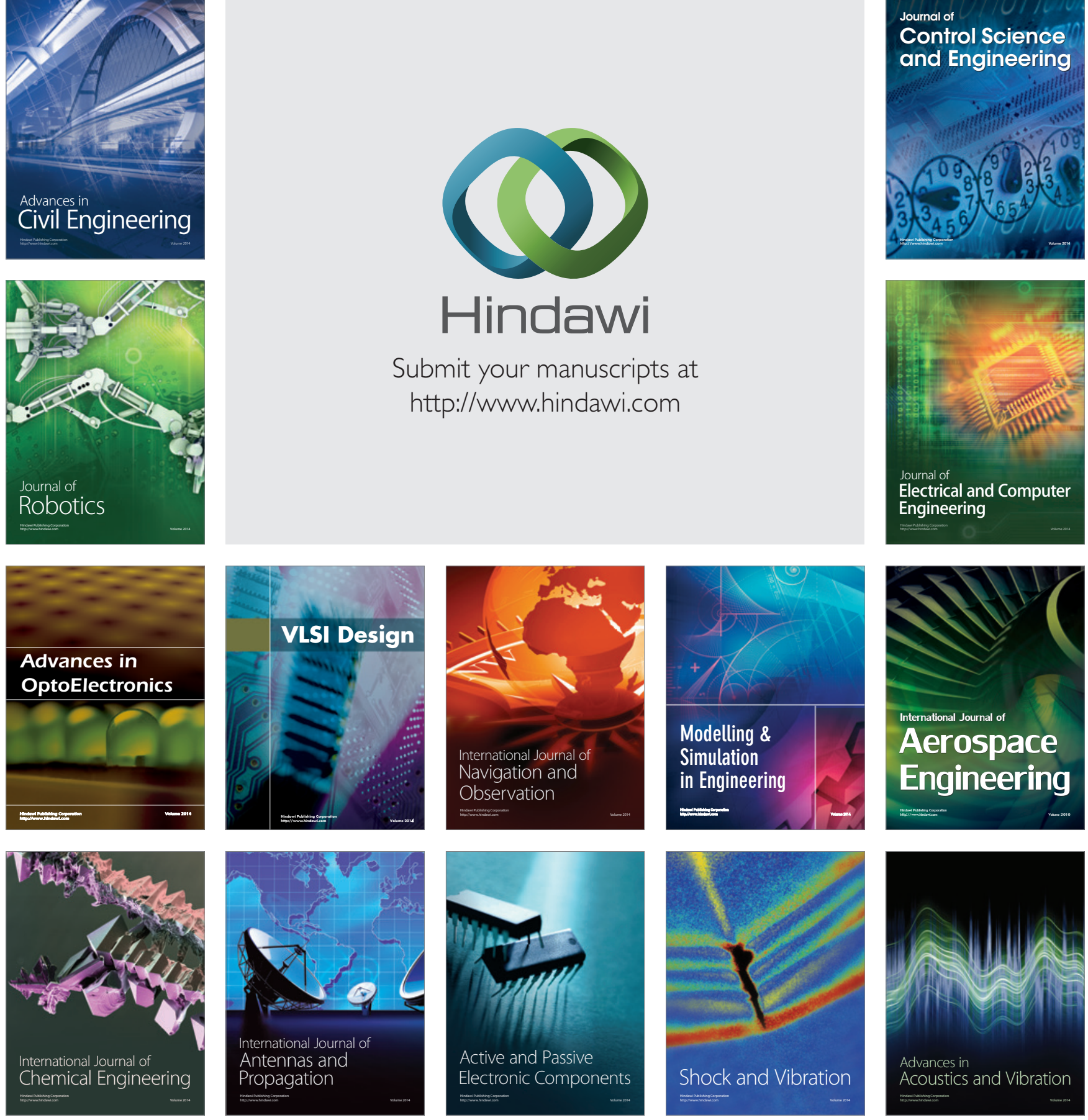